\title{
Evaluating and Differentiating a Polynomial Using a Pseudo-witness Set
}

\author{
Jonathan D. Hauenstein and Margaret H. Regan ${ }^{(凶)}$ \\ Department of Applied and Computational Mathematics and Statistics, \\ University of Notre Dame, Notre Dame, USA \\ $\{$ hauenstein, mregan9\} @nd.edu

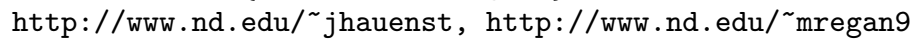

\begin{abstract}
Polynomials which arise via elimination can be difficult to compute explicitly. By using a pseudo-witness set, we develop an algorithm to explicitly compute the restriction of a polynomial to a given line. The resulting polynomial can then be used to evaluate the original polynomial and directional derivatives along the line at any point on the given line. Several examples are used to demonstrate this new algorithm including examples of computing the critical points of the discriminant locus for parameterized polynomial systems.
\end{abstract}

Keywords: Numerical algebraic geometry - Pseudo-witness set • Implicit polynomial $\cdot$ Directional derivatives $\cdot$ Critical points

\section{Introduction}

Parameterized polynomial systems arise in various applications in science and engineering, such as in computer vision [15,17,22], kinematics [14,23], and chemistry $[1,19]$. Often in these applications, real solutions are desired. The complement of the discriminant locus associated with the parameterized polynomial system consists of cells where the number of real solutions is constant. Elimination methods (e.g., see [8, Chap.3]) theoretically provide an approach to explicitly compute a defining equation for the discriminant locus. If the discriminant locus is a curve or surface, there are several numerical methods that can be used to plot it, e.g., $[6,7,18]$. When the explicit expression is difficult to compute, this paper develops a numerical algebraic geometric approach based on pseudo-witness sets [13] for both evaluating implicitly defined polynomials and directional derivatives. In particular, the approach yields an explicit univariate polynomial equal to the defining equation restricted to a line which can then be evaluated or differentiated as needed. When the parameterized system and line have rational coefficients, the resulting univariate polynomial also has rational coefficients which can be computed exactly from the numerical data [2].

One application of this new approach is to compute the critical points of the discriminant polynomial which are outside of the discriminant locus without explicitly computing the discriminant. This set of critical points contains at least

(C) Springer Nature Switzerland AG 2020

A. M. Bigatti et al. (Eds.): ICMS 2020, LNCS 12097, pp. 61-69, 2020.

https://doi.org/10.1007/978-3-030-52200-1_6 
one point in each compact cell in the complement of the discriminant locus [10] which can be useful for determining the possible number of real solutions as well as the real monodromy structure [11].

The remainder of the paper is as follows. Section 2 describes the approach based on using pseudo-witness sets. Section 3 presents an algorithm for performing the computations with some illustrative examples. Section 4 provides two examples of computing critical points.

\section{Implicit Representation of a Polynomial}

In numerical algebraic geometry, e.g., see [4,21], a witness point set for a hypersurface $\mathcal{H} \subset \mathbb{C}^{n}$ consists of the intersection points of $\mathcal{H}$ with a line $\mathcal{L} \subset \mathbb{C}^{n}$. Suppose that $f(x)$ is a given polynomial and $\mathcal{H}$ is the hypersurface defined by the vanishing of $f$. Then, the witness point set for $\mathcal{H}$ corresponds with the roots of the univariate polynomial obtained by restricting $f$ to the line $\mathcal{L}$. Since every univariate polynomial is defined up to scale by its roots, one can recover $\left.f\right|_{\mathcal{L}}$ by computing its roots along with knowing $\left.f\right|_{\mathcal{L}}(T)$ for some value $T$ which is not a root of $\left.f\right|_{\mathcal{L}}$. The following is an illustration of this basic setup.

Example 1. Consider the polynomial $f(x, y)=y-x^{2}$ with corresponding hypersurface $\mathcal{H} \subset \mathbb{C}^{2}$ and the line $\mathcal{L} \subset \mathbb{C}^{2}$ defined parametrically by:

$$
x(t)=t \quad y(t)=2 t+1 .
$$

Therefore, one can explicitly compute

$$
\left.f\right|_{\mathcal{L}}(t)=f(x(t), y(t))=-t^{2}+2 t+1=-(t-1+\sqrt{2})(t-1-\sqrt{2}) .
$$

For $t_{1}=1-\sqrt{2}$ and $t_{2}=1+\sqrt{2}$, one has

$$
\mathcal{H} \cap \mathcal{L}=\left\{\left(t_{1}, 2 t_{1}+1\right),\left(t_{2}, 2 t_{2}+1\right)\right\} .
$$

Hence, $\left.f\right|_{\mathcal{L}}(t)=s\left(t-t_{1}\right)\left(t-t_{2}\right)$ for some constant $s$ which can be computed from, say, requiring $\left.f\right|_{\mathcal{L}}(T)=1$ where $T=2$, i.e., $s=-1$. Therefore, one has recovered $\left.f\right|_{\mathcal{L}}(t)$ in $(1)$ from $\mathcal{H} \cap \mathcal{L}$ with $\left.f\right|_{\mathcal{L}}(T)=1$ as illustrated in Fig. 1(a).

The remainder of this section extends this idea using pseudo-witness sets when $f$ is a polynomial over $\mathbb{C}$ that is not known explicitly, but the corresponding hypersurface $\mathcal{H}$ arises as the closure of a projection of an algebraic set. For simplicity of presentation, assume that $F: \mathbb{C}^{N} \rightarrow \mathbb{C}^{r}$ is a polynomial system and that $V$ is a pure $d$-dimensional subset of $\mathcal{V}(F)=\left\{x \in \mathbb{C}^{N} \mid F(x)=0\right\}$. Let $\pi\left(x_{1}, \ldots, x_{N}\right)=\left(x_{1}, \ldots, x_{n}\right)$ such that $\mathcal{H}=\overline{\pi(V)} \subset \mathbb{C}^{n}$. Note that one has $n-1 \leq d \leq N-1$. A pseudo-witness set [13] for $\mathcal{H}$, say $\{F, \pi, \mathcal{M}, W\}$, is a numerical algebraic geometric data structure that permits computations on $\mathcal{H}$ without knowing the defining polynomial $f$ for $\mathcal{H}$. The last two items are a linear space $\mathcal{M} \subset \mathbb{C}^{N}$ and a finite set $W=V \cap \mathcal{M}$. In particular, $\mathcal{M}=\mathcal{L} \times \mathcal{L}^{\prime}$ where 


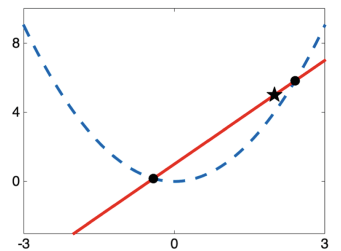

(a)

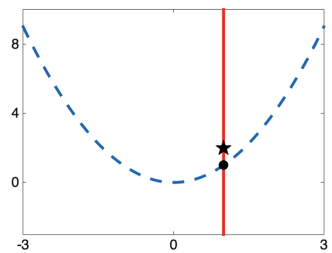

(b)

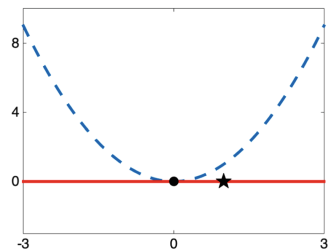

(c)

Fig. 1. A visual representation of the pseudo-witness set for $\mathcal{H}$ defined by $y-x^{2}$ with a linear slice, $\mathcal{L}$, that is (a) generic, (b) special with one root of multiplicity one, and (c) tangent. The black dots represent the roots $t_{1}, \ldots, t_{k}$ and the black stars represent $T$ selected for scale.

$\mathcal{L}^{\prime} \subset \mathbb{C}^{N-n}$ is a codimension $d-(n-1)$ general linear space so that $\mathcal{M}$ has codimension $d$. Hence, $\pi(W)=\mathcal{H} \cap \mathcal{L}$ is a witness point set for $\mathcal{H}$ with respect to $\mathcal{L}$. With this setup, the local multiplicity of each point in $\mathcal{H} \cap \mathcal{L}$ can be easily computed via [5, Prop. 6] (see also [9, pg. 158]). Thus, parameterizing $\mathcal{L}$ by $t$ and denoting $t_{1}, \ldots, t_{k}$ as the corresponding points in $\mathcal{H} \cap \mathcal{L}$ with multiplicity $m_{1}, \ldots, m_{k}$, respectively, yields

$$
\left.f\right|_{\mathcal{L}}(t)=\frac{\left.f\right|_{\mathcal{L}}(T)}{\left(T-t_{1}\right)^{m_{1}} \cdots\left(T-t_{k}\right)^{m_{k}}} \cdot\left(t-t_{1}\right)^{m_{1}} \cdots\left(t-t_{k}\right)^{m_{k}}
$$

as shown in the following.

Theorem 1. The univariate polynomial describing $f$ along the line $\mathcal{L}$ is correctly described by (3).

Proof. The assumption on $T$ is that $\left.f\right|_{\mathcal{L}}(T) \neq 0$, i.e., $\mathcal{L} \not \subset \mathcal{H}$. Hence, $\left.f\right|_{\mathcal{L}}$ is a nonzero polynomial which has finitely many roots, namely $t_{1}, \ldots, t_{k}$ with multiplicity $m_{1}, \ldots, m_{k}$, respectively. Thus, $m_{i} \geq 1$ with $\operatorname{deg}\left(\left.f\right|_{\mathcal{L}}\right)=m_{1}+\cdots+m_{k}$. Since the roots define the univariate polynomial up to scale, the leading coefficient is used to achieve the desired value at $T$ and thus everywhere along $\mathcal{L}$.

The following illustrates a pseudo-witness set and Theorem 1.

Example 2. Consider the hypersurface $\mathcal{H} \subset \mathbb{C}^{2}$ from Example 1 under the assumption that we are given $\mathcal{H}=\overline{\pi(V)}$ where $\pi(x, y, s)=(x, y)$ and $V=\mathcal{V}(F)$ with

$$
F(x, y, s)=\left[\begin{array}{l}
x-s^{2} \\
y-s^{4}
\end{array}\right] .
$$

Since $n=2$ and $d=\operatorname{dim} V=1$, we have $\mathcal{M}=\mathcal{L} \times \mathbb{C}$ with

$$
W=V \cap \mathcal{M}=\left\{\left(t_{1}, 2 t_{1}+1, \pm \sqrt{t_{1}}\right),\left(t_{2}, 2 t_{2}+1, \pm \sqrt{t_{2}}\right)\right\}
$$

where $t_{1}$ and $t_{2}$ are as in Example 1 with $m_{1}=m_{2}=1$. Hence, $\pi(W)=\mathcal{H} \cap \mathcal{L}$ as in (2). Therefore, with $T=2$ and $\left.f\right|_{\mathcal{L}}(T)=1$, (3) simplifies to $\left.f\right|_{\mathcal{L}}(t)$ in (1). 
The only assumption on the line $\mathcal{L}$ is that $\mathcal{L} \not \subset \mathcal{H}$ so that one can find $T$ such that $\left.f\right|_{\mathcal{L}}(T) \neq 0$. Of course, one can check if $\mathcal{L} \subset \mathcal{H}$ by a pseudo-witness set membership test [12] in which case one would simply have $f_{\mathcal{L}}(t) \equiv 0$. Thus, $\mathcal{L}$ is not necessarily assumed to intersect $\mathcal{H}$ transversely, so the number of roots and multiplicities can vary for different choices of $\mathcal{L}$. Nonetheless, Theorem 1 applies as is illustrated in the following two examples.

Example 3. Reconsider Example 2 with $\mathcal{L}$ being the vertical line parametrized by

$$
x(t)=1 \quad y(t)=t
$$

as shown in Fig. 1(b). One has $\mathcal{M}=\mathcal{L} \times \mathbb{C}$ and $W=V \cap \mathcal{M}=\{(1,1, \pm 1)\}$ with $t_{1}=1$ and $m_{1}=1$. For scale, consider $T=2$ with $\left.f\right|_{\mathcal{L}}(T)=1$. Thus, (3) yields

$$
\left.f\right|_{\mathcal{L}}(t)=t-1
$$

Example 4. Reconsider Example 2 with $\mathcal{L}$ being the horizontal line parametrized by

$$
x(t)=t \quad y(t)=0
$$

as shown in Fig. $1(\mathrm{c})$. One has $\mathcal{M}=\mathcal{L} \times \mathbb{C}$ and $W=V \cap \mathcal{M}=\{(0,0,0)\}$ with $t_{1}=0$ and $m_{1}=2$. For scale, consider $T=1$ with $\left.f\right|_{\mathcal{L}}(T)=-1$. Thus, (3) yields

$$
\left.f\right|_{\mathcal{L}}(t)=-t^{2}
$$

Clearly, once the univariate polynomial $\left.f\right|_{\mathcal{L}}(t)$ in $(3)$ is computed explicitly, one can easily determine the value of $f$ at any point along $\mathcal{L}$ via evaluation. Moreover, if $\mathcal{L}$ is parameterized by $v \cdot t+u$, then $\left.f\right|_{\mathcal{L}} ^{(k)}(t)$ is equal to the $k^{\text {th }}$ directional derivative of $f$ with respect to $v$ at $v \cdot t+u$, denoted $D_{v}^{(k)} f(v \cdot t+u)$.

Example 5. For $\mathcal{L}$ in Example 3 and Example 4, one has $v=(0,1)$ and $v=$ $(1,0)$, respectively. Hence, the corresponding directional derivatives are simply partial derivatives of $f(x, y)=y-x^{2}$ with respect to $y$ and $x$, respectively. From Example 3, one obtains $\frac{\partial f}{\partial y}(1, t)=1$ while Example 4 yields $\frac{\partial f}{\partial x}(t, 0)=-2 t$ and $\frac{\partial^{2} f}{\partial x^{2}}(t, 0)=-2$.

\section{Algorithm}

Theorem 1 immediately justifies Algorithm 1 for explicitly computing a polynomial restricted to a line. The following two examples exemplify this algorithm applied to the discriminant locus.

Example 6. Consider the discriminant locus $\mathcal{H} \subset \mathbb{C}^{2}$ for $g(x ; b, c)=x^{2}+b x+c$. Hence, $\mathcal{H}=\overline{\pi(V)}$ where $\pi(b, c, x)=(b, c)$ and $V=\mathcal{V}(F)$ with

$$
F(b, c, x)=\left[\begin{array}{c}
x^{2}+b x+c \\
2 x+b
\end{array}\right] .
$$


Algorithm 1. Computing a polynomial restricted to a line

Input: A line $\mathcal{L} \subset \mathbb{C}^{n}$ parameterized by $t$, a pseudo-witness set $\{F, \pi, \mathcal{M}, W\}$ for a hypersurface $\mathcal{H}$ defined by $f$ such that $\mathcal{M}=\mathcal{L} \times \mathcal{L}^{\prime}$, and $T$ along with $\left.f\right|_{\mathcal{L}}(T) \neq 0$.

Output: The univarite polynomial $\left.f\right|_{\mathcal{L}}(t)$ corresponding to $f$ restricted to $\mathcal{L}$.

1: Use the pseudo-witness set to extract the roots $t_{1}, \ldots, t_{k}$ of $f$ along $\mathcal{L}$ and the corresponding multiplicities $m_{1}, \ldots, m_{k}$.

2: Compute the scale factor $s:=\frac{\left.f\right|_{\mathcal{L}}(T)}{\left(T-t_{1}\right)^{m_{1}} \cdots\left(T-t_{k}\right)^{m_{k}}}$.

3: Construct the univariate polynomial $\left.f\right|_{\mathcal{L}}(t):=s \cdot\left(t-t_{1}\right)^{m_{1}} \cdots\left(t-t_{k}\right)^{m_{k}}$.

4: (Optional) If $\mathcal{L}$ and $F$ are defined with rational coefficients and $T$ and $\left.f\right|_{\mathcal{L}}(T)$ are rational, expand $\left.f\right|_{\mathcal{L}}(t)$ and use exactness recovery [2] to compute the exact rational coefficients of $\left.f\right|_{\mathcal{L}}(t)$.

5: Return $\left.f\right|_{\mathcal{L}}(t)$.

For the line $\mathcal{L} \subset \mathbb{C}^{2}$ parameterized by

$$
b(t)=t \quad c(t)=t / 3
$$

with $\mathcal{M}=\mathcal{L} \times \mathbb{C}$, one has $W=V \cap \mathcal{M}=\{(0,0,0),(4 / 3,4 / 9,-2 / 3)\}$. The other input for Algorithm 1 is, say, $T=3$ with $\left.f\right|_{\mathcal{L}}(T)=5$ to set the scale. This setup is illustrated in Fig. 2(a).

The pseudo-witness set yields $t_{1}=0$ and $t_{2}=4 / 3$ with $m_{1}=m_{2}=1$. The corresponding scale factor is

$$
s=\frac{5}{(3-0)(3-4 / 3)}=1
$$

so that Algorithm 1 returns $\left.f\right|_{\mathcal{L}}(t)=t(t-4 / 3)=t^{2}-4 t / 3$.

Of course, one can easily compute that the discriminant of $g$ satisfying $f(b(T), c(T))=5$ is $f(b, c)=b^{2}-4 c$ with $\left.f\right|_{\mathcal{L}}(t)=f(b(t), c(t))=t^{2}-4 t / 3$.

Example 7. Consider the discriminant locus $\mathcal{H} \subset \mathbb{C}^{2}$ for $g(x)=x^{3}+b x+c$. Hence, $\mathcal{H}=\overline{\pi(V)}$ where $\pi(b, c, x)=(b, c)$ and $V=\mathcal{V}(F)$ with

$$
F(b, c, x)=\left[\begin{array}{c}
x^{3}+b x+c \\
3 x^{2}+b
\end{array}\right] \text {. }
$$

For the line $\mathcal{L} \subset \mathbb{C}^{2}$ parameterized by

$$
b(t)=t \quad c(t)=t+3
$$

with $\mathcal{M}=\mathcal{L} \times \mathbb{C}$, one has, rounded to 4 decimal places with $i=\sqrt{-1}$,

$$
W=V \cap \mathcal{M}=\left\{\begin{array}{c}
(-1.9511,1.0489,0.8064) \\
(-2.3995 \pm 5.0378 i, 0.6005 \pm 5.0378 i,-1.1532 \pm 0.7281 i)
\end{array}\right\} .
$$

The other input for Algorithm 1 is, say, $T=-3$ with $\left.f\right|_{\mathcal{L}}(T)=-108$ for scale. This setup is illustrated in Fig. 2(b). 
The pseudo-witness set yields $t_{1}=-1.9511, t_{2}=-2.3995+5.0378 i$, and $t_{3}=-2.3995-5.0378 i$ with $m_{1}=m_{2}=m_{3}=1$. The corresponding scale factor is $s=4$ so that $\left.f\right|_{\mathcal{L}}(t)=4 t^{3}+27 t^{2}+162 t+243$.

As in Example 6, one can easily compute that the discriminant of $g$ satisfying $f(b(T), c(T))=-108$ is $f(b, c)=4 b^{3}+27 c^{2}$ with $f(b(t), c(t))=\left.f\right|_{\mathcal{L}}(t)$ as above.

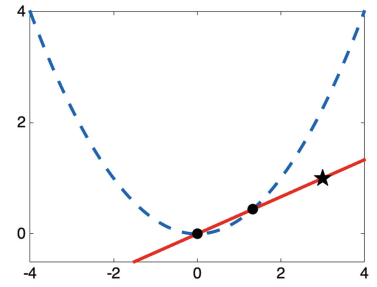

(a)

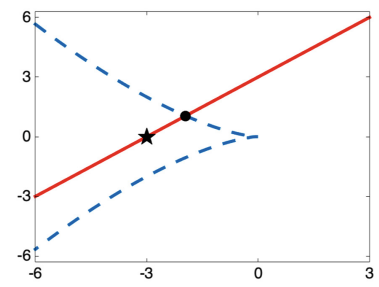

(b)

Fig. 2. Pseudo-witness set for the discriminant locus of (a) the quadratic $x^{2}+b x+c$ and (b) the cubic $x^{3}+b x+c$.

\section{Computing Critical Points}

When the line $\mathcal{L}$ is fixed, Algorithm 1 computes the restriction of a polynomial $f$ to $\mathcal{L}$. The following presents two examples of combining this idea with homotopy continuation to compute critical points of $f$, namely $\nabla f=0$. The set of real solutions to $\nabla f=0$ with $f \neq 0$ contains at least one point in each compact cell of $\mathbb{R}^{n} \cap\{f \neq 0\}$ [10]. The website dx.doi.org/10.7274/r0-0mc0-gt33 contains the necessary files to perform these computations using Bertini [3].

\subsection{Lemniscate}

This first example demonstrates the approach given $f(x, y)=x^{4}-x^{2}+y^{2}$ which defines a lemniscate, but utilizes a pseudo-witness set for the computation. The aim is to compute all real solutions of $\nabla f=0$ and $f \neq 0$. For genericity, replace $\nabla f=0$ with the equivalent condition that the directional derivatives of $f$ in both the $\alpha=\left(\alpha_{1}, \alpha_{2}\right)$ and $\beta=\left(\beta_{1}, \beta_{2}\right)$ directions, namely $D_{\alpha} f$ and $D_{\beta} f$, vanish for general $\alpha$ and $\beta$. We used $\alpha_{1}=1, \alpha_{2}=5+3 \sqrt{-1}, \beta_{1}=4+\sqrt{-1}$, and $\beta_{2}=1$ in our computation.

Since one is setting directional derivatives equal to zero, the scale factor is irrelevant and can be simply set to 1 . We first compute a witness set for each of the cubic curves defined by $D_{\alpha} f=0$ and $D_{\beta} f=0$ where each of them are expressed in terms of univariate roots following Sect. 2 . Then, we simply intersect these two cubic curves using a diagonal homotopy [20] that tracks $3^{2}=9$ paths. There are 3 finite endpoints corresponding with the 3 solutions of $\nabla f=0$, all of which are real and shown in Fig. 3(a). Two of these have $f \neq 0$ with one in each of the two compact cells of $\mathbb{R}^{2} \cap\{f \neq 0\}$. 


\subsection{Kuramoto Model}

The Kuramoto model [16] is a mathematical model of an oscillating system to describe synchronization. After a standard conversion to polynomials, the discriminant locus for the steady states of the 3-oscillator Kuramoto model is modeled by $\mathcal{H}=\overline{\pi(V)}$ where $\pi\left(\omega_{1}, \omega_{2}, c_{1}, c_{2}, s_{1}, s_{2}\right)=\left(\omega_{1}, \omega_{2}\right)$ and $V=\mathcal{V}(F)$ with

$$
F\left(\omega_{1}, \omega_{2}, c_{1}, c_{2}, s_{1}, s_{2}\right)=\left[\begin{array}{c}
\left(s_{1} c_{2}-c_{1} s_{2}\right)+\left(s_{1} c_{3}-c_{1} s_{3}\right)-3 \omega_{1} \\
\left(s_{2} c_{1}-c_{2} s_{1}\right)+\left(s_{2} c_{3}-c_{2} s_{3}\right)-3 \omega_{2} \\
s_{1}^{2}+c_{1}^{2}-1 \\
s_{2}^{2}+c_{2}^{2}-1 \\
c_{1}^{2} c_{2}+c_{1} c_{2}^{2}+c_{1} c_{2}+s_{1} s_{2} c_{1}+s_{1} s_{2} c_{2}
\end{array}\right] .
$$

Letting $f$ be a defining polynomial for $\mathcal{H}$, the aim is to compute the real solutions of $\nabla f=0$ with $f \neq 0$ using a pseudo-witness set for $\mathcal{H}$. As in Sect. 4.1, we replace $\nabla f=0$ with the equivalent condition that two general directional derivatives vanish. In this case, the vanishing of a general directional derivative of $f$ yields a degree 11 curve, so a diagonal homotopy [20] to intersect the vanishing of two directional derivatives tracks $11^{2}=121$ paths. This yields 103 finite solutions consisting of 37 that satisfy $f \neq 0$ which can be verified using a membership test via a pseudo-witness set for $\mathcal{H}$ [12]. Sorting through these 37 yields 19 real critical points with $f \neq 0$. Figure $3(\mathrm{~b})$ plots the real part of $\mathcal{H}$ along with these 19 real critical points on a contour plot of $f$ showing that at least one is contained in each compact cell of $\mathbb{R}^{2} \cap\{f \neq 0\}$.

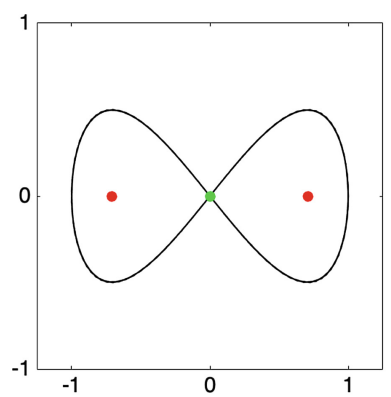

(a)

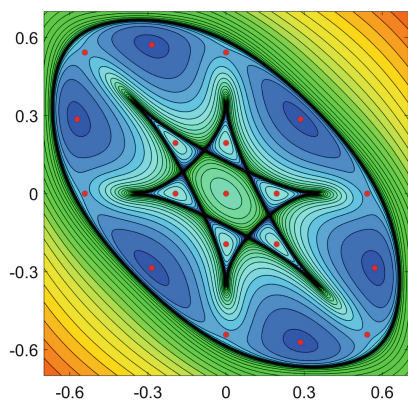

(b)

Fig. 3. (a) The lemniscate with 2 critical points satisfying $f \neq 0$ (red) and the other satisfying $f=0$ (green), and (b) the discriminant locus (black) for the 3-oscillator Kuramoto model with contour plot and 19 real critical points (red) in the complement. (Color figure online)

Acknowledgments. All authors acknowledge support from NSF CCF-1812746. Additional support for was provided by ONR N00014-16-1-2722 (Hauenstein) and Schmitt Leadership Fellowship in Science and Engineering (Regan). 


\section{References}

1. Al-Khateeb, A.N.: One-dimensional slow invariant manifolds for spatially homogeneous reactive systems. J. Chem. Phys. 131 (2009)

2. Bates, D.J., Hauenstein, J.D., McCoy, T.M., Peterson, C., Sommese, A.J.: Recovering exact results from inexact numerical data in algebraic geometry. Exp. Math. 22(1), 38-50 (2013)

3. Bates, D.J., Hauenstein, J.D., Sommese, A.J., Wampler, C.W.: Bertini: software for numerical algebraic geometry. bertini.nd.edu

4. Bates, D.J., Hauenstein, J.D., Sommese, A.J., Wampler, C.W.: Numerically solving polynomial systems with Bertini. SIAM (2013)

5. Bernardi, A., Daleo, N.S., Hauenstein, J.D., Mourrain, B.: Tensor decomposition and homotopy continuation. Differ. Geom. Appl. 55, 78-105 (2017)

6. Besana, G.M., Di Rocco, S., Hauenstein, J.D., Sommese, A.J., Wampler, C.W.: Cell decomposition of almost smooth real algebraic surfaces. Numer. Algor. 63, 645-678 (2013)

7. Chen, C., Wu, W.: A numerical method for computing border curves of biparametric real polynomial systems and applications. In: Gerdt, V.P., Koepf, W., Seiler, W.M., Vorozhtsov, E.V. (eds.) CASC 2016. LNCS, vol. 9890, pp. 156-171. Springer, Cham (2016). https://doi.org/10.1007/978-3-319-45641-6_11

8. Cox, D.A., Little, J., O'Shea, D.: Ideals, Varieties, and Algorithms, 4th edn. Springer, Cham (2015). https://doi.org/10.1007/978-3-319-16721-3

9. Fischer, G.: Complex Analytic Geometry. Lecture Notes in Mathematics, vol. 538. Springer, Heidelberg (1976). https://doi.org/10.1007/BFb0080338

10. Harris, K., Hauenstein, J.D., Szanto, A.: Smooth points on semi-algebraic sets. arXiv:2002.04707 (2020)

11. Hauenstein, J.D., Regan, M.H.: Real monodromy action. Appl. Math. Comput. 373, 124983 (2020)

12. Hauenstein, J.D., Sommese, A.J.: Membership tests for images of algebraic sets by linear projections. Appl. Math. Comput. 219(12), 6809-6818 (2013)

13. Hauenstein, J.D., Sommese, A.J.: Witness sets of projections. Appl. Math. Comput. 217(7), 3349-3354 (2010)

14. Hauenstein, J.D., Wampler, C.W., Pfurner, M.: Synthesis of three-revolute spatial chains for body guidance. Mech. Mach. Theory 110, 61-72 (2017)

15. Irschara, A., Zach, C., Frahm, J.M., Bischof, H.: From structure-from-motion point clouds to fast location recognition. In: CVPR, pp. 2599-2606. IEEE (2009)

16. Kuramoto, Y.: Self-entrainment of a population of coupled non-linear oscillators. In: Araki, H. (ed.) International Symposium on Mathematical Problems in Theoretical Physics. Lecture Notes in Physics, vol. 39, pp. 420-422. Springer, Heidelberg (1975). https://doi.org/10.1007/BFb0013365

17. Leibe, B., Cornelis, N., Cornelis, K., Van Gool, L.: Dynamic 3D scene analysis from a moving vehicle. In: CVPR, pp. 1-8. IEEE (2007)

18. Lu, Y., Bates, D.J., Sommese, A.J., Wampler, C.W.: Finding all real points of a complex curve. Contemp. Math. 448, 183-205 (2007)

19. Morgan, A.P.: Solving Polynomial Systems Using Continuation for Engineering and Scientific Problems. Prentice-Hall, Englewood Cliffs (1987)

20. Sommese, A.J., Verschelde, J., Wampler, C.W.: Solving polynomial systems equation by equation. In: Dickenstein, A., Schreyer, F.O., Sommese, A.J. (eds.) Algorithms in Algebraic Geometry. The IMA Volumes in Mathematics and its Applications, vol. 146, pp. 133-152. Springer, New York (2008). https://doi.org/10.1007/ 978-0-387-75155-9_8 
21. Sommese, A.J., Wampler, C.W.: The Numerical Solution of Systems of Polynomials Arising in Engineering and Science. World Scientific, Hackensack (2005)

22. Snavely, N., Seitz, S.M., Szeliski, R.: Photo tourism: exploring photo collections in 3D. In: ACM SIGGRAPH, pp. 835-846 (2006)

23. Wampler, C.W., Sommese, A.J.: Numerical algebraic geometry and algebraic kinematics. Acta Numerica 20, 469-567 (2011) 